\title{
PiRNA-DQ541777 Contributes to Neuropathic Pain via Targeting Cdk5rap1
}

\author{
Chenjing Zhang, ${ }^{1 \star}$ Huanhuan Sha, ${ }^{2 \star}$ Yunan Peng, ${ }^{3 \star}$ Yin Wang, ${ }^{4}$ Cunming Liu, ${ }^{2}$ and $\odot$ Xuelong Zhou ${ }^{2}$ \\ ${ }^{1}$ Department of Gastroenterology, Zhejiang Province People’s Hospital, Hangzhou, Zhejiang 310014, China, ${ }^{2}$ Department of Anesthesiology, the First \\ Hospital of Nanjing Medical University, Nanjing, Jiangsu 210029, China, ${ }^{3}$ Department of Anesthesiology, Affiliated Drum Tower Hospital of Nanjing \\ University Medical School, Nanjing, Jiangsu 210008, China, and ${ }^{4}$ Department of Anesthesiology, Taizhou People's Hospital, Taizhou, Jiangsu 225300, China
}

Piwi-Interacting RNA (piRNA) is the largest class of small noncoding RNA and is involved in various physiological and pathological processes. However, whether it has a role in pain modulation remains unknown. In the present study, we found that spinal piRNADQ541777 (piR-DQ541777) was significantly increased in the male mouse model of sciatic nerve chronic constriction injury (CCI)induced neuropathic pain. Knockdown of spinal piR-DQ541777 alleviated CCI-induced thermal hyperalgesia and mechanical allodynia and spinal neuronal sensitization. However, the overexpression of spinal piR-DQ541777 in naive mice produced pain behaviors and increased spinal neuron sensitization. Furthermore, we found that piR-DQ541777 regulates pain behaviors by targeting CDK5 regulatory subunit-associated protein 1 (Cdk5rap1). CCI increased the methylation level of CpG islands in the $c d k 5$ rapl promoter and consequently reduced the expression of Cdk5rap1, which was reversed by the knockdown of piR-DQ541777 and mimicked by the overexpression of piR-DQ541777 in naive mice. Finally, piR-DQ541777 increased the methylation level of CpG islands by recruiting DNA methyltransferase $3 \mathrm{~A}$ (DNMT3a) to $c d k 5 \mathrm{rap} 1$ promoter. In conclusion, this study represents a novel role of piR-DQ541777 in the regulation of neuropathic pain through the methylation of $c d k 5 r a p 1$.

Key words: neuronal sensitization; neuropathic pain; Piwi-interacting RNA; spinal cord

\section{Significance Statement}

Chronic pain affects $\sim 20 \%$ of the population of the world and is a major global public health problem. Although we have studied the neurobiological mechanism of neuropathic pain for decades, there is still no ideal drug available to treat it. This work indicates that a novel role of Piwi-interacting RNA (piRNA) DQ541777 in the regulation of neuropathic pain through the methylation of $c d k 5$ rap1. Our findings provide the first evidence of the regulatory effect of piRNAs on neuropathic pain, which may improve our understanding of pain mechanisms and lead to the discovery of novel drug targets for the prevention and treatment of neuropathic pain.

\section{Introduction}

Noncoding RNA (ncRNA) is a class of RNA that normally does not encode a protein, including, for example, microRNA, small interfering RNA (siRNA), long-noncoding RNA, and circle RNA. At present, increasing evidence shows that ncRNA plays an important role in the modulation of a diversity of physiological and pathophysiological conditions, such as tumor generation, neurodevelopment, and nervous system diseases (Esteller, 2011). Piwi-

Received July 7, 2019; revised Aug. 26, 2019; accepted Sept. 3, 2019.

Author contributions: Y.P., C.L., and X.Z. designed research; C.Z., H.S., Y.P., and Y.W. performed research; C.Z., H.S., Y.P., Y.W., and X.Z. analyzed data; X.Z. wrote the paper.

This work was supported by grants from the National Natural Science Foundation of China (Grant 81701101).

${ }^{*}$ C.Z., H.S., and Y.P. contributed equally to this work

The authors declare no competing financial interests.

Correspondence should be addressed to Xuelong Zhou at xhjxlj@sina.cn.

https://doi.org/10.1523/JNEUROSCI.1602-19.2019

Copyright $\odot 2019$ the authors interacting RNAs (piRNAs) are a novel class of small non-coding RNAs that were first discovered in mouse germ line cells (Vagin et al., 2006). They are $\sim 24-33 \mathrm{nt}$ in length, slightly longer than microRNA (22 nt). piRNA exerts its function by specifically binding to the Piwi subfamily of the Argonaute protein family (Iwasaki et al., 2015). Recent studies have found that piRNA is essential for germline stem cell maintenance, transcriptional gene silencing, and mRNA translation and stability (Iwasaki et al., 2015; Ozata et al., 2019). The discovery of piRNA opens up a new field for the study of ncRNA. Further study of its biological functions will deepen our understanding of specific disease pathophysiological processes.

Previous studies have shown that piRNA is widely expressed in the central and peripheral nervous system and participates in regulating various neurophysiological and neuropathological processes (Lee et al., 2011; Phay et al., 2018). For example, piR-F in Aplysia brain involved in the maintenance of long-term mem- 
ory (Rajasethupathy et al., 2012). Knockdown of specific piRNA in mouse hippocampus neurons reduced dendritic spines formation (Lee et al., 2011). Interestingly, Phay et al., 2018 recently found that peripheral nerve injury caused significant changes in piRNA expression in rat sciatic nerve axoplasm, suggesting that piRNA may be associated with the regulation of nociceptive processing (Phay et al., 2018). PiRNA-DQ541777 (piR-DQ541777) is highly expressed in the mammalian CNS and is involved in the modulation of synaptic plasticity (Lee et al., 2011). Given the essential role of synaptic plasticity in chronic pain (Kuner and Flor, 2016), we hypothesized that piR-DQ541777 may be involved in the modulation of pain information processing. In the present study, using a chronic constriction injury (CCI) of the sciatic nerve model, we examined the correlation between the expression of piR-DQ541777 and neuropathic pain and the molecular mechanism of how piR-DQ541777 modulates neuropathic pain. This study is one of the first to directly demonstrate a role for piRNAs in neuropathic pain, which suggests that piRNAs may be a novel target for future therapeutic development.

\section{Materials and Methods}

Animal pain models. All experiments were conducted in accordance with the animal protection and use policies of the International Association for the Study of Pain and Nanjing Medical University. Adult male C57BL/6 mice were used in this experiment. Mice were kept in a $12 \mathrm{~h}$ light/dark cycle with temperature controlled at $22-26^{\circ} \mathrm{C}$ and provided with food and water ad libitum. Neuropathic pain was induced by ligation of the right sciatic nerve (CCI model; Bennett and Xie, 1988), unilateral ligation of the L5 and L6 spinal nerves [spinal nerve ligation (SNL) model; Kim and Chung, 1992], and axotomy and ligation of the tibial and common peroneal nerves [spared nerve injury (SNI) model; Decosterd and Woolf, 2000]. For sham-operated mice, the nerve was exposed but without ligation or transection. Inflammatory pain was induced by intraplantar injection of complete Freund's adjuvant (CFA) into the plantar surface or formalin into the dorsal surface of the mouse hindpaw. Normal saline was used as a control.

Behavior tests. Mice were placed in a plastic box on the glass plate and subjected to behavioral tests after $1 \mathrm{~h}$ of habituation. A radiant heat source was focused onto the plantar surface of the hindpaw. The time taken to elicit a withdrawal response (a lifting or licking of the hindpaw) was recorded as paw withdrawal latency (PWL; Hargreaves et al., 1988). The base value was adjusted to $12-15 \mathrm{~s}$, and an automatic $25 \mathrm{~s}$ cutoff was set to prevent burning injury. The test was performed three times with an interval of $5 \mathrm{~min}$. For the paw withdrawal threshold (PWT), mice were placed on a metal mesh floor in a plastic box. A series of von Frey filaments (starting with $0.31 \mathrm{~g}$ and ending with $4.0 \mathrm{~g}$ ) were applied to stimulate the hindpaw plantar surface. A positive response was considered if the mice exhibited a brisk withdrawal or paw flinching. Results were analyzed using the Dixon up-and-down method (Chaplan et al., 1994). All the behavioral tests were carried out in a blinded manner.

Reverse transcription-quantitative PCR. Total RNA was extracted from mouse spinal cords using RNeasy Mini Kit, SurePrep Small RNA Purification Kit, or SurePrep Nuclear or Cytoplasmic RNA Purification Kit. The concentrations and quality were determined by the NanoDrop 2000. The purified RNA was reverse transcribed into cDNA using the SuperscriptIII First-strand synthesis system. Real-time quantitative PCR (RT-qPCR) was performed using SYBR Green PCR Master Mix. Data were analyzed using the $\Delta \Delta \mathrm{Ct}$ method (Livak and Schmittgen, 2001). U6 RNA and $\beta$-actin were used as internal control genes. The primers used for RT-qPCR were as follows: piR-DQ541777 RT: 5'-GTCGTATCCA GTGCAGGGTCCGAGGTATTCGCACTGGATACGACTTGAGA-3'; piR-DQ541777 S: 5'-GCTGGTCCGAAGGTAGTGA-3'; piR-DQ541777 AS: 5'-GCAGGGTCCGAGGTTATTC-3'; cdk5rapl S: 5'-CCATGTGCT GGGTGTTGCTTA-3'; $c d k 5$ rap 1 AS: $5^{\prime}$ - TCTGCCTTCCTAGAAGTTC ATCC-3'; gapdh S: 5' -GGCTGTATTCCCCTCCATCG-3'; gapdh AS: 5' CCAGTTGGTAACAATGCCATGT-3'; U6 S: 5'-CTCGCTTCGGCAG
CACATATACT-3'; and U6 AS: 5'-ACGCTTCACGAATTTGCGT GTC-3'.

Lentivirus production and intrathecal injection. The short hairpin RNAs (shRNAs) specifically targeting piR-DQ541777 and Cdk5rap1 were designed and inserted into the pSIH1-Puro-GFP lentiviral shRNA vector (sh-DQ541777, sh-Cdk5rap1). A scrambled shRNA was used as a control. In addition, the sequence of piR-DQ541777 or $c d k 5$ rapl was inserted into the lentiviral vector pLent-Puro-GFP (LV-DQ541777, LVCdk5rap1). An empty pLent-Puro-GFP vector served as a negative control. Intrathecal injection was performed using a 28 gauge stainless steel needle. A sudden slight flick of the tail after insertion between the L5 and L6 vertebrae indicated entry into the subarachnoid space, and $8 \times 10^{5}$ transduction unites (TU) virus was injected each time. The knockdown or overexpression of piR-DQ541777 in the spinal cord was verified by RT-qPCR.

RNA-binding protein immunoprecipitation. RNA was isolated from spinal cords using the RNA-Binding Protein Immunoprecipitation (RIP) Kit according to the manufacturer protocol. Spinal cords were lysed in complete RIP lysis buffer. Ten microliters of the supernatant of RIP lysate was removed and place into a new tube that was labeled "input." This input sample was stored at $-80^{\circ} \mathrm{C}$ until RNA purification. Five micrograms of the antibody [anti-miwi, anti-DNA methyltransferase 1 (DNMT1), anti-DNMT3a, anti-DNMT3b, or normal rabbit IgG] was added to the tube and incubated with rotation for $30 \mathrm{~min}$ at room temperature. The beads were washed twice. All the tubes contained cell lysate and antibody and beads were incubated with rotating for $4 \mathrm{~h}$ to overnight at $4^{\circ} \mathrm{C}$. Next, the beads were washed with cold RIP Wash Buffer. Twenty microliters of the beads were removed and placed into a new tube for Western blot. Then, all tubes were incubated with proteinase $\mathrm{K}$ at $55^{\circ} \mathrm{C}$ for $30 \mathrm{~min}$. The supernatant was transferred into a new tube. Finally, the RNA was extracted and analyzed by RT-qPCR.

Fluorescence in situ hybridization, immunofluorescence, and immunohistochemistry. Mice were anesthetized followed by a sternotomy surgery. Tissues were fixed by intracardial perfusion with $4 \%$ paraformaldehyde. Spinal cord tissues were isolated, postfixed with $4 \%$ paraformaldehyde, and then dehydrated with $30 \%$ sucrose solution. The prepared spinal cord was cut into $30-\mu \mathrm{m}$-thick sections. Digoxigenin-labeled lock nucleic acid-based antisense oligonucleotides, as well as sense oligonucleotide probes, were synthesized. Fluorescence in situ hybridization (FISH) was performed using FISH detection kit, and then FISH sections were incubated with NeuN antibody or Cdk5rap1 antibody, finally incubated with fluorescent-conjugated secondary antibody. Fos immunohistochemistry was performed as we have previously reported (Zhou et al., 2017).

Immunoblotting. Spinal cords were quickly dissected from anesthetized mice. The spinal cord tissues were collected and homogenized in lysis buffer containing protease inhibitor. The homogenates were centrifuged, and the supernatant was collected. Protein concentrations were measured using the BCA Protein Assay Kit. Protein samples were separated using SDS-PAGE and transferred onto a polyvinylidene difluoride membrane. The membranes were incubated with the primary antibodies overnight at $4^{\circ} \mathrm{C}$. After extensive washing, the membranes were incubated with horseradish peroxidase-conjugated secondary antibody for $2 \mathrm{~h}$ at room temperature. The immune complexes were detected by using a chemiluminescence detection kit. The signal intensity was analyzed using ImageJ software. Immunoprecipitations were carried out using Catch and Release version 2.0 Reversible Immunoprecipitation System Kit according to the manufacturer instructions.

Bisulfite sequencing. Mouse genomic DNA was isolated by the QIAamp DNA Mini Kit. Purified DNA was subjected to bisulfite conversion using the EZ DNA Methylation-Gold Kit. Bisulfite-modified DNAs were amplified by PCR using the following primers: $\mathrm{CpG}$ island1-S1, 5'-AAAGT TAGGGTTTTGGGATT-3'; CpG island1-AS1, 5'-AACCCCATCTCT ACTTCACAAAAC-3'; CpG island2-S2, 5' - GTTTTGTGAAGTAGAG ATGGGGT-3'; and CpG island2-AS1, 5'-ACTAACTACTTTTCCA AAACCTAAAT- $3^{\prime}$. The DNA fragment was gel purified using the QIAquick Gel Extraction Kit followed by cloned into pGEM-T Vector and sequenced. 
Dual-luciferase reporter assay. The defined region of piR-DQ541777 promoter was amplified from mouse genomic DNA (pGL-C1-S: 5'AGAAGTAGTAGGTCTCACGGCT-3', pGL-C1-AS: $5^{\prime}$-TCACAGGG CAAATACTAGGGC-3'; pGL-C2-S: 5'-GCCCTAGTATTTGCCCTGT GAA-3', pGL-C2-AS: 5'-AGCAGCCCACACTTCATTTC-3'; pGL-C1/ 2-S: 5'-AAGAAGTAGTAGGTCTCACGGC-3'; and pGL-C1/2-AS: 5'ACTAGCAGCCCACACTTCAT-3') and cloned into a pGL6 plasmid (pGL-C1 contains the $\mathrm{CpG}$ island 1, pGL-C2 contains the CpG island 2, pGL-C1/2 contains both the two CpG islands). Empty pGL6 vector was used as a control plasmid. pGL-Cs or pGL-control was methylated using methyltransferase (M.SssI) at $37^{\circ} \mathrm{C}$ for $1 \mathrm{~h}$. Activities of luciferase were measured at $48 \mathrm{~h}$ after transfection of plasmids using the dual-luciferase reporter assay system kit. The binding sites between piR-DQ541777 and cdk5rap1 were modified through site-directed mutagenesis using QuikChange Site-Directed Mutagenesis Kit.

Chromatin immunoprecipitation. Chromatin immunoprecipitation (ChIP) assays were performed using the SimpleChIP Enzymatic Chromatin IP Kit according to the manufacturer instructions. Briefly, spinal tissues were cross-linked with $1 \%$ formaldehyde followed by adding the ChIP lysis buffer with protease inhibitors. The tissue was sonicated to obtain chromatin with an average size of 200-500 bp. Immunoprecipitation was incubated with anti-DNMT1, anti-DNMT3a, and antiDNMT3b antibodies followed by immunoprecipitation with Protein G Agarose Beads in each sample during an overnight incubation at $4^{\circ} \mathrm{C}$. Normal rabbit IgG was used as a control. Meanwhile, $2 \%$ input reference was removed and stored at $-80^{\circ} \mathrm{C}$ before adding antibody. The ChIP DNA cross-link was reversed by $5 \mathrm{~mol} / \mathrm{L} \mathrm{NaCl}$ and Proteinase $\mathrm{K}$ at $65^{\circ} \mathrm{C}$ for $2 \mathrm{~h}$. Immunoprecipitated DNA was amplified by PCR using their specific primers. In each PCR, the corresponding input was taken in parallel for PCR validation.

Experimental design and statistical analysis. All mice were male, and the experimental groups were age matched. The sample size was based on the results of preliminary data and previous experiments. All data were presented as the mean $\pm \mathrm{SD}$. Comparison between two groups was performed using the unpaired $t$ test. Comparison between more than two groups was analyzed using one-way ANOVA followed by a Tukey's post hoc test. For repeated-measurement data (behavioral data), two-way ANOVA with Bonferroni's post hoc test was used to do the statistical analysis. All the comparisons were conducted using PRISM 5 software. $p<0.05$ was considered to be statistically significant.

\section{Results}

\section{PiRNA-DQ541777 was increased in the spinal cord in CCI mice}

To investigate whether piR-DQ541777 is involved in pain modulation, we first induced neuropathic pain in mice using sciatic CCI surgery. Behavioral results revealed that strong thermal hyperalgesia and mechanical hyperalgesia developed on day 1 , peaked on day 3, and persisted over $14 \mathrm{~d}$ after CCI (Fig. 1A). Spinal tissues were then collected, and the time course of spinal piR-DQ541777 expression was evaluated. The results from RTqPCR showed that piR-DQ541777 level was gradually increased from day 1 to day 3 after CCI and was maintained at the peak for at least $14 \mathrm{~d}$ (Fig. 1B). Similar results were also observed in two other neuropathic pain models. Neuropathic pain induced by SNI and SNL significantly increased the expression of spinal piRDQ541777 on day 7 after surgery when compared with the sham groups (Fig. 1C). Moreover, we found that the spinal piRDQ541777 level was considerably increased in the CFA-induced chronic inflammatory pain (on day 3 after injection; Fig. 1D). However, no significant alteration of spinal piR-DQ541777 was found in the formalin-induced acute inflammatory pain model (at $2 \mathrm{~h}$ after injection; Fig. 1D), implying that the upregulation of spinal piR-DQ541777 may be involved in the development of chronic pain rather than acute pain. Next, we used a FISH experiment to further confirm an increase in piR-DQ541777 expres- sion in the spinal dorsal horn of CCI mice compared with that of the sham group. Additionally, FISH and immunofluorescence costaining showed that piR-DQ541777 was colocalized with NeuN (a marker of neuronal cell bodies; Fig. 1E). piRNAs are associated with Piwi proteins to form an effector multiprotein complex. In mouse, Piwi clade members are MIWI, MILI, and MIWI2. However, only MIWI forms a functional complex with piRNA in the nervous system (Lee et al., 2011; Phay et al., 2018). To test whether piR-DQ541777 is complexed with the Piwi proteins, we used a MIWI antibody to immunoprecipitate the piRNAs in mice. The results from RIP revealed that piRDQ541777 interacted with MIWI protein and that this interaction level was increased in the CCI group versus the sham group (Fig. $1 F$ ). Finally, subcellular fractionation location assays revealed the localization of piR-DQ541777 to both in the nucleus and cytoplasm (Fig. 1G). Together, these findings suggest that piRDQ541777 was increased in the spinal cord during neuropathic pain conditions.

\section{Manipulation of piR-DQ541777 in the spinal cord modulates pain behaviors and spinal neuronal sensitization}

To determine the role of spinal piR-DQ541777 in the regulation of neuropathic pain, the following two tools were used in the present study: shRNA lentivirus was used to silence piRDQ541777 expression; and the overexpression lentivirus was used to upregulate piR-DQ541777 expression.

The transfection efficiency of siRNA or lentivirus was validated in the spinal cord of CCI or naive mice by RT-qPCR (Fig. $2 A, E$ ). First, intrathecal injection of piR-DQ541777 shRNA (shDQ541777) for $2 \mathrm{~d}$ before CCI (pretreatment) significantly alleviated CCI-induced thermal hyperalgesia and mechanical allodynia on days $1,3,5$, and 7 (Fig. 2B). Moreover, shDQ541777 was intrathecally injected daily for $2 \mathrm{~d}$ on days 5 and 6 after CCI (post-treatment) and significantly reversed CCIinduced thermal hyperalgesia and mechanical allodynia from day 7 to day 9 (Fig. 2C). Peripheral noxious stimuli or injury trigger Fos protein expression in the spinal cord, which has been used extensively as a marker of neuronal sensitization related to pain regulation (Gao and Ji, 2009). To investigate the effect of knockdown of piR-DQ541777 on CCI-induced spinal neuronal sensitization, we examined the expression of Fos protein in the spinal cord after sh-DQ541777 administration. The results from immunohistochemistry showed that either pretreatment or posttreatment with sh-DQ541777 significantly inhibited the CCIinduced increase in spinal Fos expression (Fig. 2D), suggesting that piR-DQ541777 silencing-induced alleviation of neuropathic pain may occur through the inhibition of spinal neuronal sensitization. Next, we examined whether the overexpression of piRDQ541777 by piR-DQ541777 lentivirus (LV-DQ541777) could induce pain hypersensitivity in naive mice. Intrathecal injection of LV-DQ541777, not its negative control lentivirus (LV-NC), into naive mice daily for $2 \mathrm{~d}$ significantly produced thermal hyperalgesia and mechanical allodynia (Fig. $2 F$ ). Furthermore, spinal administration of LV-DQ541777 also increased spinal Fos expression (Fig. 2F). These findings suggest that piR-DQ541777 in the spinal cord is essential for neuropathic pain regulation.

\section{PiRNA-DQ541777 regulates CCI-induced neuropathic pain by targeting Cdk5rap1}

To identify potential targets of piR-DQ541777, BLAST (Basic Local Alignment Search Tool) alignment was performed on-line. The piRNA target-binding sites search is based on sequence complementarity. In addition, RNAup (http://rna.tbi.univie.ac.at/ 

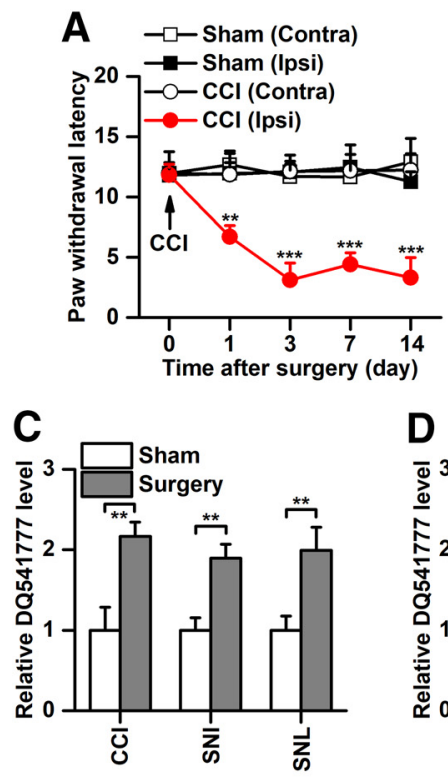
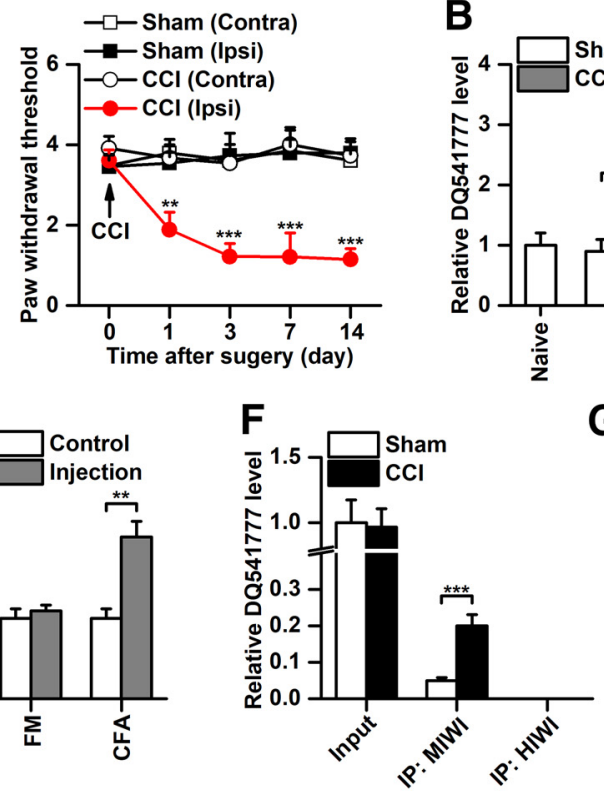

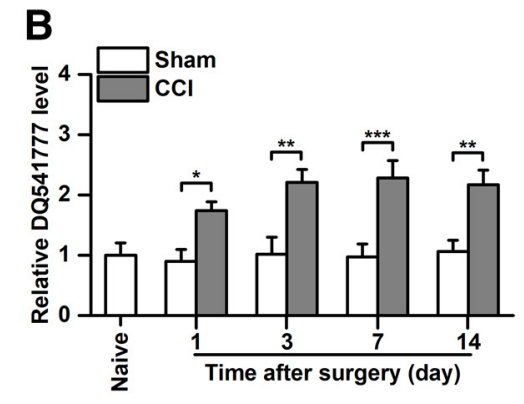

G

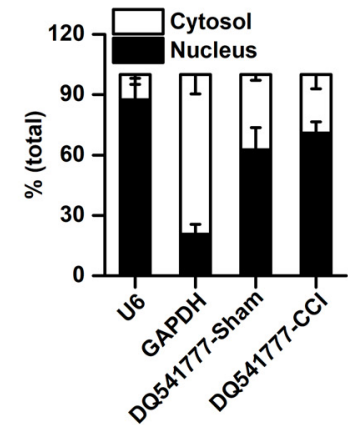

$\mathbf{E}$
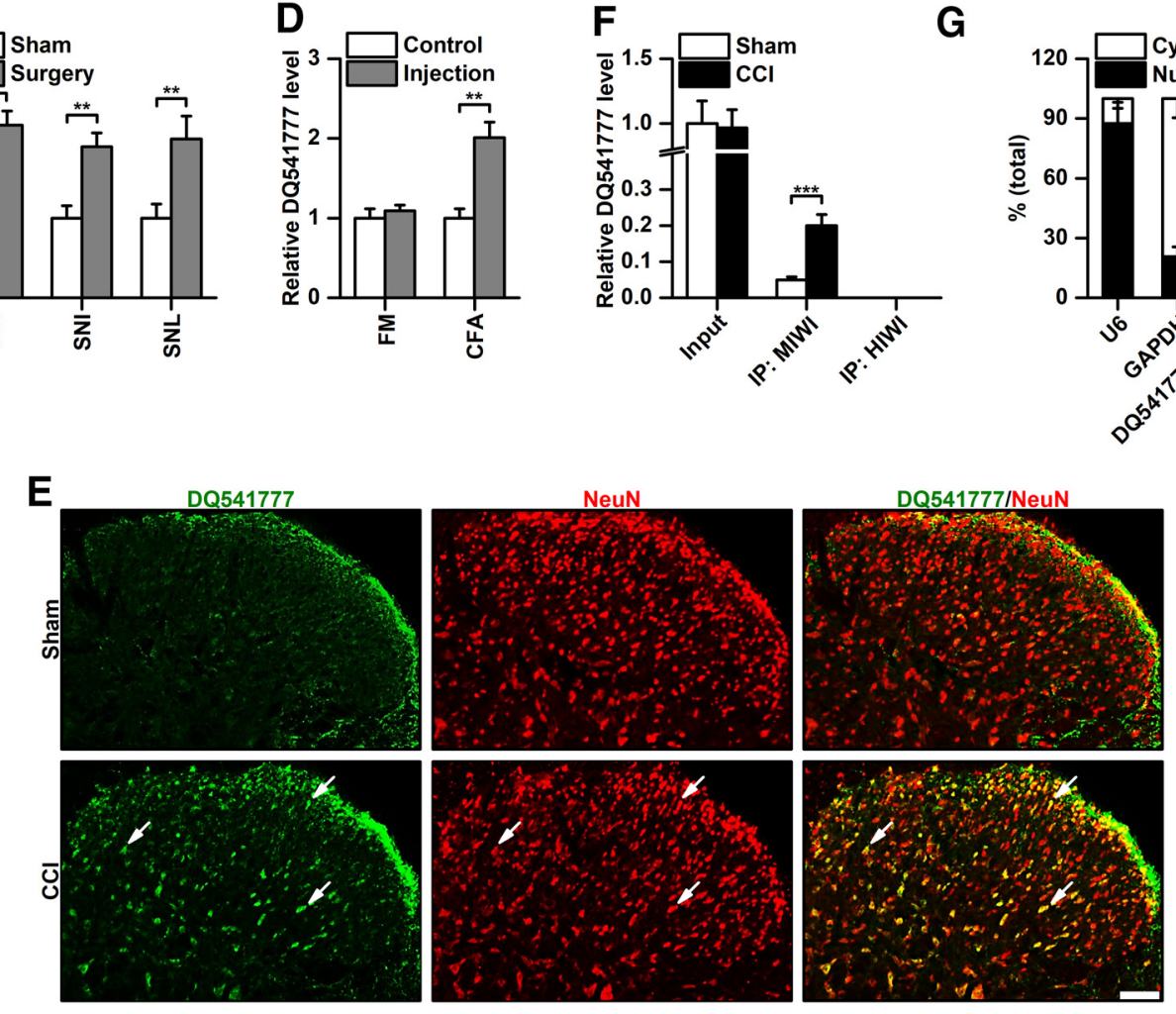

Figure 1. PiR-DQ541777 expression in the spinal cord. A, Alterations of nociceptive behaviors after CCI. Two-way ANOVA; group for PWL, $F_{(3,28)}=221.06, p<0.001 ;$ group for PWT, $F_{(3,28)}=257.3, p<0.001 ;{ }^{* *} p<0.01,{ }^{* * *} p<0.001$, CCI-Ipsi vs CCI-control. Ipsi, Ipsilateral hindpaw; Contral, contralateral hindpaw; $n=8$. $B$, Time course of spinal piR-DQ541777 (DQ541777) expression in CCl-induced neuropathic pain. One-way ANOVA; $F_{(4,24)}=14.793, p<0.01 ;{ }^{*} p<0.05,{ }^{* *} p<0.01,{ }^{* * *} p<0.001, n=6$. C, Spinal piR-DQ541777 expression on day 7 after $\mathrm{CCl}$, SNI, and SNL surgery. $t_{(10)}=8.523,9.463,7.176 ;{ }^{* *} p<0.01, n=6$. D, Spinal piR-DQ541777 expression at $2 \mathrm{~h}$ after formalin injection and $3 \mathrm{~d}$ after $\mathrm{CFA}$ injection. $t_{(10)}=10.94{ }^{* *} p<0.01, n=6$. E, PiR-DQ541777 FISH and NeuN immunofluorescence staining in the spinal cord on day 7 after CCl or sham surgery. Scale bar, $25 \mu \mathrm{m}$. Arrow indicates the representative examples of double-labeled cells. $\boldsymbol{F}, \mathrm{RT}$-qPCR expression of piR-DQ541777 after immunoprecipitation with MIWI antibody. $t_{(10)}=12.28,{ }^{* * *} p<0.001, n=6 . \mathbf{G}$, Subcellular fractionation location assays of piR-DQ541777 in the spinal cord.

cgi-bin/RNAWebSuite/RNAup.cgi) was used to calculate the free energy of the binding sites. The bioinformatical search revealed that piR-DQ541777 could target several neuronal mRNAs at a low free energy level, including Cdk5rap1 (Fig. 3A) and Mark1/2. As Cdk5rap1 is the endogenous inhibitor of CDK5 (Ching et al., 2002; Liu et al., 2018), which is a key player in pain information processing in the spinal cord (Fang-Hu et al., 2015; Moutal et al., 2019), we chose to study its potential role as a target of piRDQ541777 in neuropathic pain regulation. To experimentally validate our bioinformatics predictions, we first examined the expression level of Cdk5rapl in the spinal cord after CCI. The results of RT-qPCR and Western blot showed that CCI, not sham control surgery, significantly decreased the expression of spinal Cdk5rap1 (Fig. 3B). The correlation analysis revealed that Cdk5rap1 showed a significant inverse correlation with piR-DQ541777 expression (Fig. 3C). Further, FISH-immunofluorescent costaining showed that Cdk5rap1 and piR-DQ541777 are coexpressed in spinal cells (Fig.
3D). Second, we quantitatively analyzed the changes of Cdk5rap1 expression after the manipulation of piR-DQ541777 in mice. The knockdown of piR-DQ541777 by its specific shRNA significantly reversed the decreased Cdk5rap1 expression in the spinal cord of CCI mice (Fig. 3E). However, lentivirus-mediated overexpression of piR-DQ541777 greatly decreased spinal Cdk5rap1 level in naive mice (Fig. 3F). Third, to explore the role of Cdk5rap1 in the mediation of pain regulation by spinal piR-DQ541777 at the behavioral level, we pretreated the mice with an shRNA to knock down Cdk5rap 1 before spinal administration of sh-DQ541777, then measured the behavioral response. The behavioral results showed that the knockdown of Cdk5rap 1 abolished the analgesia effect of sh-DQ541777 on the CCI-induced thermal hyperalgesia and mechanical allodynia (Fig. $3 G$ ). Furthermore, overexpression of Cdk5rap1 by post-treatment with LVCdk5rap1 significantly inhibited the thermal hyperalgesia and mechanical allodynia induced by LV-DQ541777 in naive 

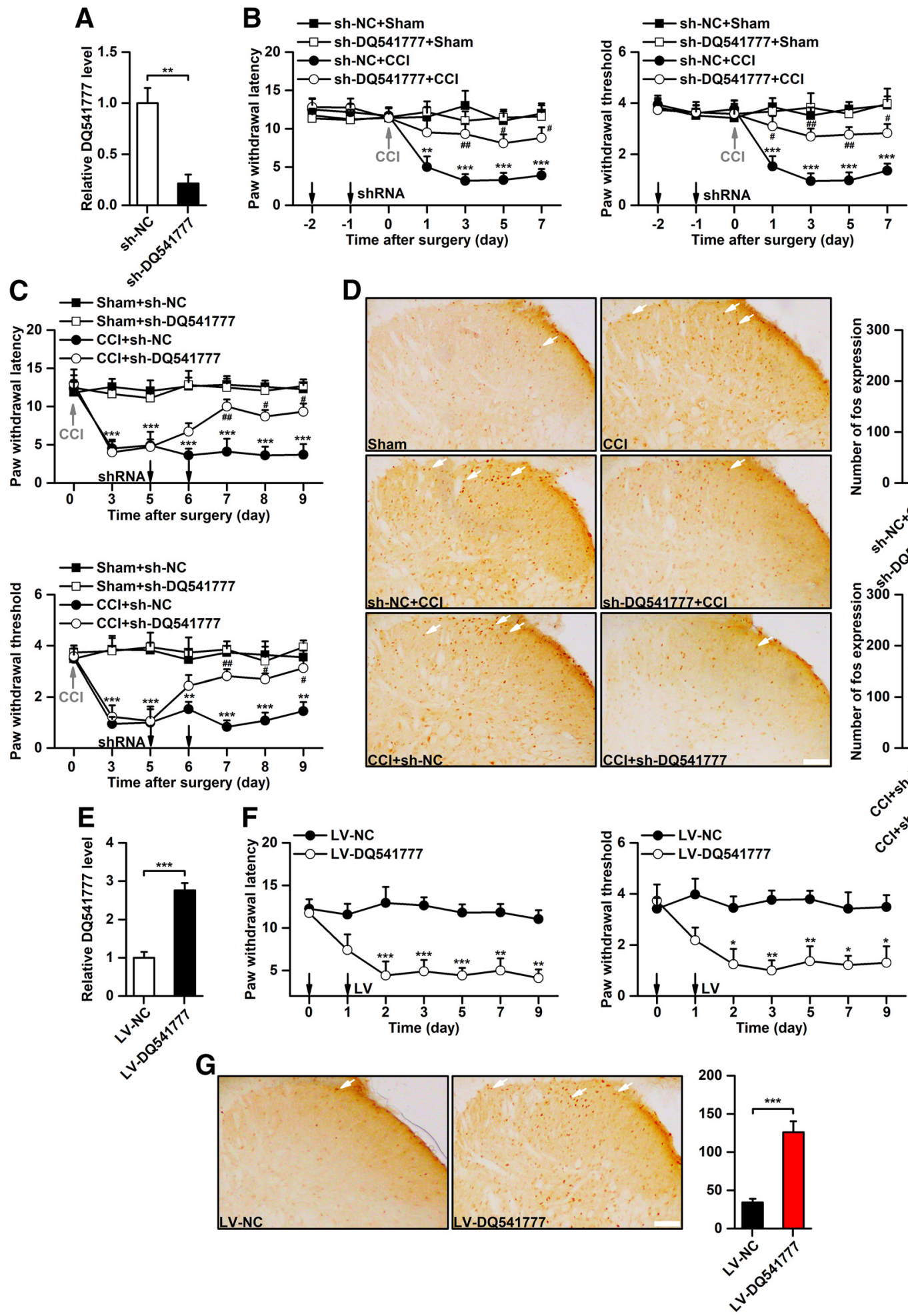

Figure 2. Spinal piR-DQ541777 regulates pain behaviors and spinal neuronal sensitization. $A$, The validation of sh-DQ541777 transfection efficiency in the spinal cord of CCI mice. $t_{(10)}=12.20$; ${ }^{* *} p<0.01, n=6$. $\boldsymbol{B}$, Changes of nociceptive behaviors after pretreatment with sh-DQ541777 in CCl mice. Two-way ANOVA; group for PWL, $F_{(3,49)}=141.9, p<0.01 ;$ group for PWT, $F_{(3,28)}=$ $173.8, p<0.001 ;{ }^{* *} p<0.01$, ${ }^{* * *} p<0.001$, sh-NC+CCl vs sh-NC+Sham; \#p $<0.01$, \#\#p $<0.001$, sh-DQ541777 +CCl vs sh-NC+CCl. NC, Negative control; $n=8$. C, Changes of nociceptive behaviors post-treatment with sh-DQ541777 in CCI mice. Two-way ANOVA; group for PWL, $F_{(3,49)}=425.2, p<0.001$; group for PWT, $F_{(3,39)}=405.4, p<0.001$; **p $<0.01$, *** $p<0.001$, $\mathrm{CCl}+$ sh-NC vs Sham + sh-NC; \#p $<0.01$, \#\#p $<0.001, \mathrm{Cl}+$ sh-DQ541777 vs $\mathrm{CCl}+$ sh-NC; $n=8$. D, Spinal Fos expression after pretreatment (on day 3) and post-treatment (on day 7) with sh-DQ541777 in C(I mice. $t_{(10)}=13.1,14.44 ;{ }^{* * *} p<0.001, n=6$. Arrows indicate the representative examples of positive Fos signal. Scale bar, $25 \mu \mathrm{m}$. $\boldsymbol{E}$, The validation of LV-DQ541777 transfection efficiency in the spinal cord of naive mice. $t_{(10)}=17.38$; ${ }^{* *} p<0.001, n=6 . F$, Changes of nociceptive behaviors after intrathecal injection of LV-DQ541777 in naive mice. Two-way ANOVA; group for PWL, $F_{(1,14)}=94.23, p<0.01$; group for PWL, $F_{(1,14)}=89.56, p<0.01 ;{ }^{*} p<0.05,{ }^{* *} p<0.01,{ }^{* * *} p<0.001, n=8$. G, Spinal Fos expression on day 3 after intrathecal injection of LV-DQ541777 in naive. $t_{(10)}=14.68 ;{ }^{* * *} p<0.001, n=6$. Arrows indicate the representative examples of positive Fos signal. Scale bar, $25 \mu \mathrm{m}$. 
A

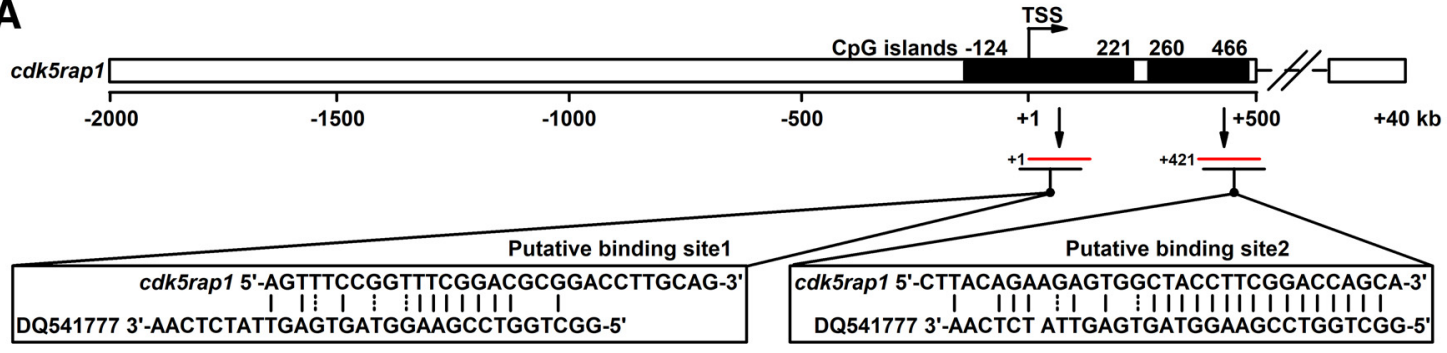

B

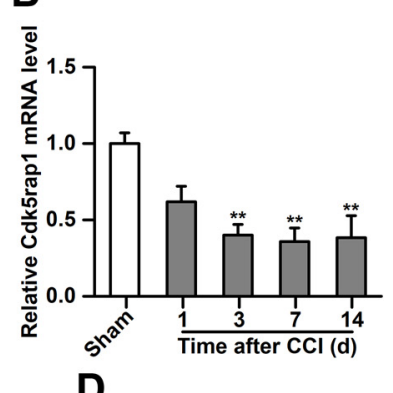

D DQ541777
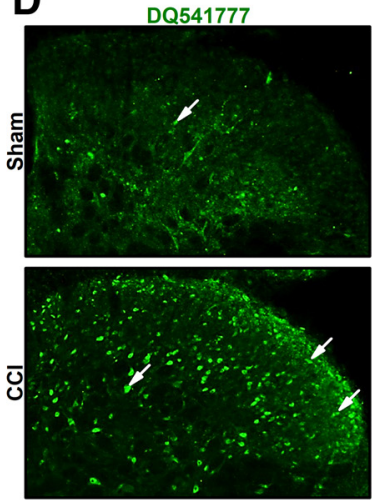

E
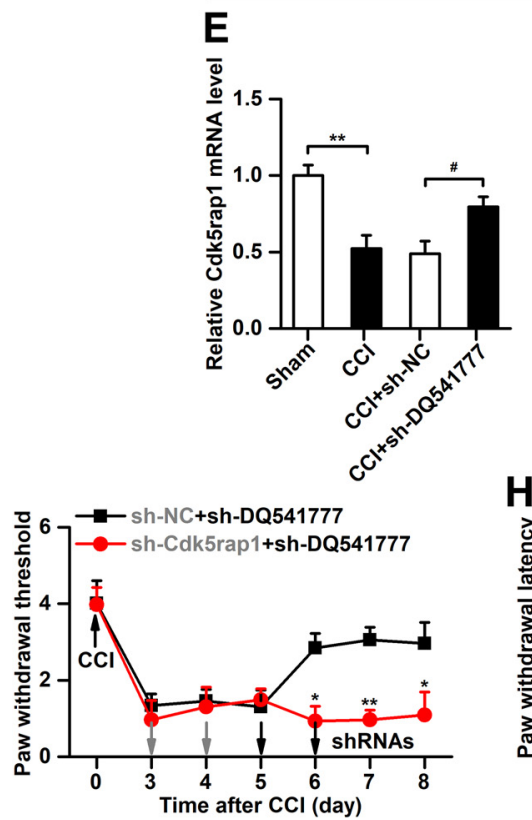
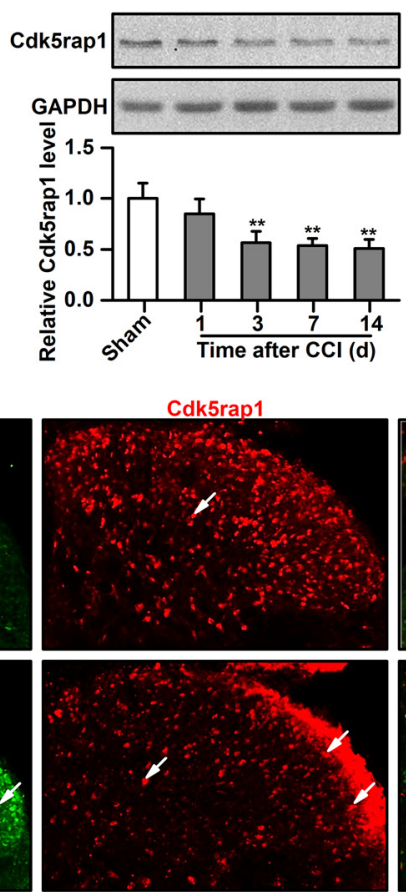

F
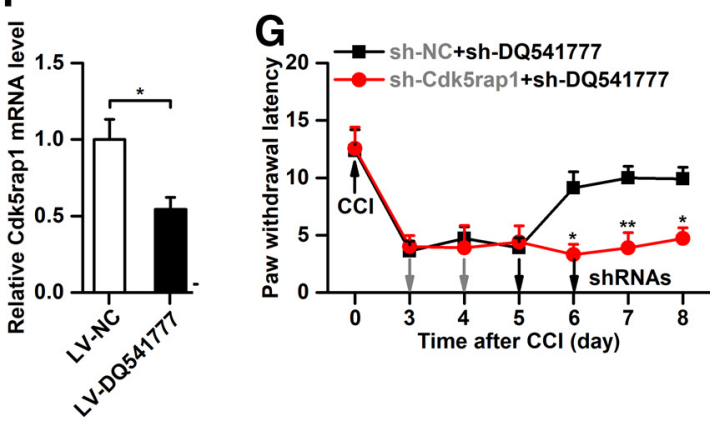

C

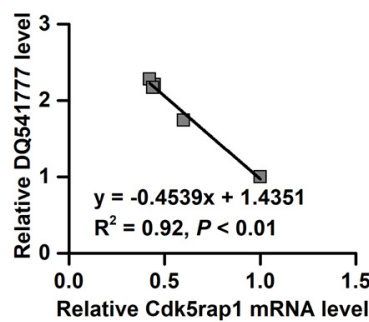

DQ541777/Cdk5rap1
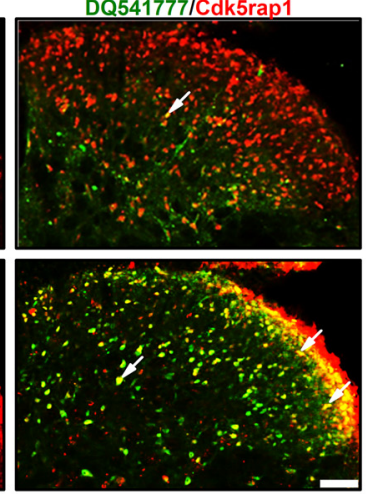

H

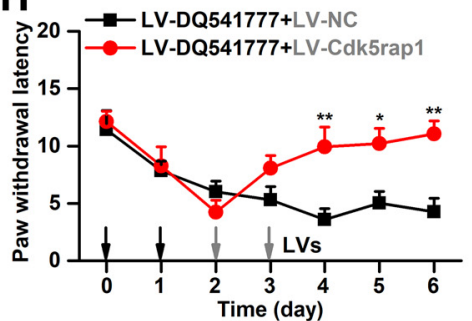

Figure 3. piR-DQ541777 regulates neuropathic pain by targeting Cdk5rap1. A, The bioinformatics search revealed that cdk5rap 1 has two putative binding sites with piR-DQ541777. TSS, Transcription start site. $\boldsymbol{B}$, Spinal Cdk5rap1 mRNA and protein expression in CCl-induced neuropathic pain. One-way ANOVA; $F_{(4,24)}=44.30,20.58, p<0.01 ;{ }^{*} p<0.0$, ${ }^{* *} p<0.01, n=6$. C, Correlation analysis of Cdk5rap1 with piR-DQ541777 in the spinal cord after CCI. D, PiR-DQ541777 FISH and Cdk5rap1 immunofluorescent costaining in the spinal cord on day 7 after CCI. Scale bar, $25 \mu \mathrm{m}$. Arrow indicates the representative examples of double-labeled cells. $\boldsymbol{E}$, Spinal Cdk5rap 1 expression on day 7 post-treatment with sh-DQ541777 in C(I mice. One-way ANOVA; $F_{(3,20)}=60.41$, $p<0.001 ;{ }^{*} p<0.05$, $\# p<0.05, n=6$.F, Spinal Cdk5rap1 expression on day 3 after intrathecal injection of LV-DQ541777 in naive mice. $t_{(10)}=7.29 ;{ }^{*} p<0.05, n=6 . G$, Knockdown of Cdk5rap1 reversed the analgesia effect of sh-DQ541777 on thermal hyperalgesia and mechanical allodynia in CCI mice. Two-way ANOVA; group for PWL, $F_{(1,14)}=48.16, p<0.01 ;$ group for PWL, $F_{(1.14)}=$ $52.65, p<0.01 ;{ }^{*} p<0.05,{ }^{* *} p<0.01, n=8 . \mathbf{H}$, Overexpression of Cdk5rap1 inhibited LV-DQ541777-induced thermal hyperalgesia and mechanical allodynia in naive mice. Two-way ANOVA; group for PWL, $F_{(1,14)}=42.43, p<0.01$; group for PWL, $F_{(1,14)}=48.1, p<0.01 ;{ }^{*} p<0.05,{ }^{* *} p<0.01, n=8$. 

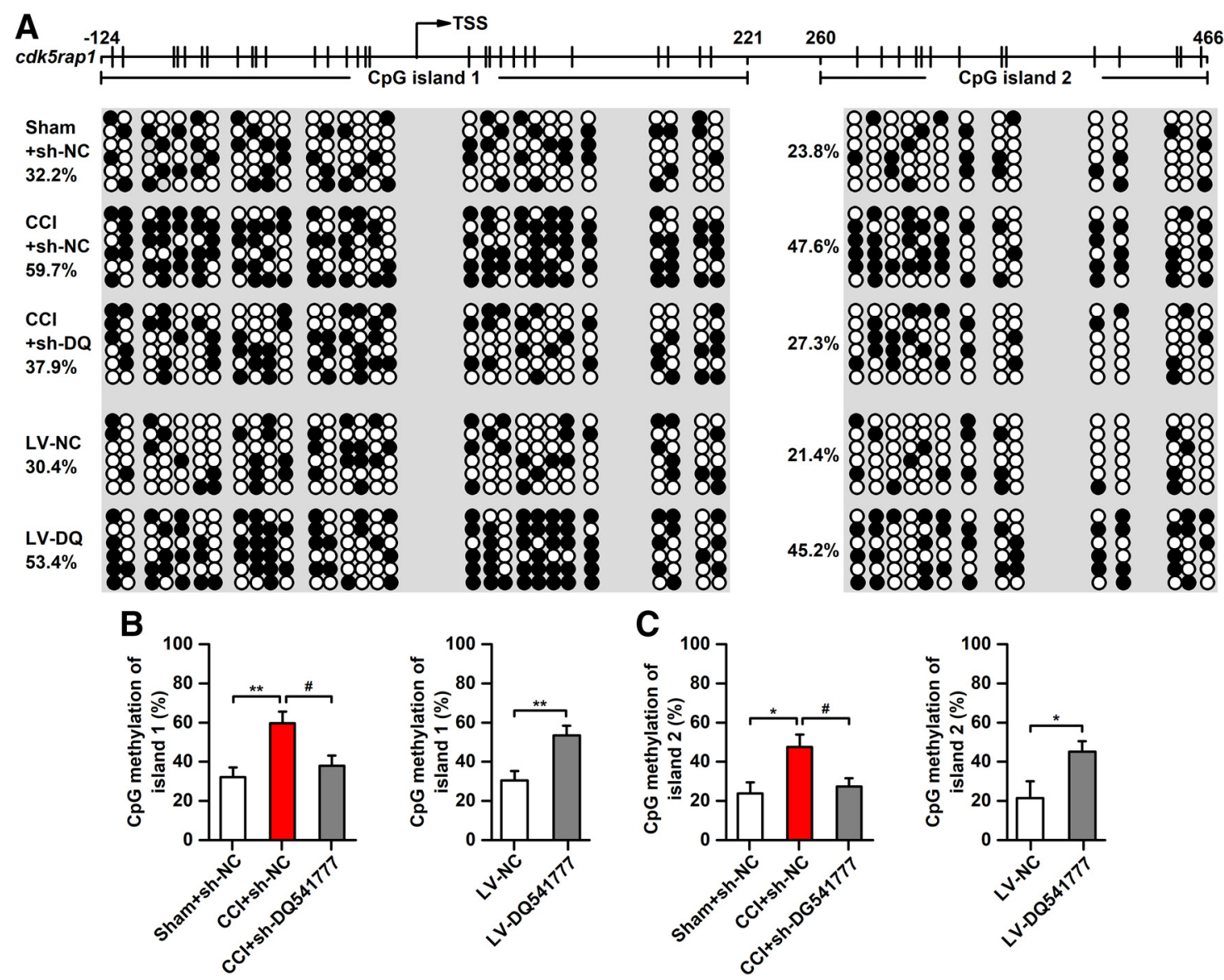

Figure 4. piR-DQ541777 regulates the C $\mathrm{pG}$ islands methylation of $c d k 5$ rap 1. A, The indication of the methylation status of C $\mathrm{pG}$ islands 1 and 2 in the $c d k 5$ rap $1 \mathrm{gene}$. Each row of circles represents a single cloned allele, and each circle indicates a single $\mathrm{CpG}$ site at a specific location. The filled and open circles represent the methylated and unmethylated $\mathrm{CpG}$ sites, respectively. $B$, Changes in the methylation level of $\mathrm{CpG}$ island 1 of the $c d k 5$ rap 1 gene on day 7 post-treatment with sh-DQ541777 in CCI mice. One-way ANOVA; $F_{(2,15)}=44.77, p<0.01$; ${ }^{* *} p<0.01, \# p<0.05, n=6$. C, Changes of the methylation level of ( $p$ G island 2 of the $c d k 5$ rap 1 gene on day 3 after intrathecal injection of LV-DQ541777 in naive mice. $t_{(10)}=8.12$; ${ }^{*} p<0.05, \# p<0.05, n=6$.

mice (Fig. 3H). Together, these findings suggest that piRDQ541777 regulates pain behaviors by targeting Cdk5rap1.

PiR-DQ541777 increases the methylation level of $c d k 5 r a p 1$ There are two potential binding sites between piR-DQ541777 and $c d k 5 \mathrm{rap} 1$, which are very close to the transcriptional initiation site of the $c d k 5 \mathrm{rap} 1$ gene (Fig. $3 A$ ). In addition, these two binding sites are located in the region of two CpG islands (CpG island 1, -124-221; CpG island 2, 260-446). Given that piRNA was proposed to guide DNA methylation and that the DNA methylation gene plays an important role in gene silence (Rajasethupathy et al., 2012; Holoch and Moazed, 2015), we hypothesized that piRDQ541777 inhibits the expression of Cdk5rap1 by regulating cdk5rap1 gene methylation. To confirm our hypothesis, we first analyzed the methylation status of the two CpG islands by bisulfite sequencing. The results showed that both $\mathrm{CpG}$ islands 1 and 2 were densely methylated in CCI mice compared with the sham group, which was inhibited by treatment with sh-DQ541777 (Fig. $4 A-C)$. In contrast, the overexpression of piRNA-DQ541777 increased the methylation levels of CpG islands 1 and 2 in naive mice (Fig. 4A-C). Next, we cloned three segments of the $c d k 5 r a p 1$ gene, including CpG island 1, CpG island 2, and both of the two CpG islands, into the upstream of the pGL6 luciferase reporter (named as pGL-C1, pGL-C2, and pGL-C-1/2, respectively) and detected their ability to drive luciferase expression in HEK293T cells. We found that all three reporter vectors produced greater luciferase activities than the control vector (Fig. 5A). However, the increased luciferase activity induced by pGL-C1 was much higher than that induced by pGL-C2 (Fig. 5A), indicating that the cloned pGL-C1 region contains the mainly regulatory element of the $c d k 5$ rap 1 gene. More importantly, methylation of pGL-C1 and pGL-C2 in vitro by the methyltransferase M.SssI before being introduced into the cells abolished their enhancement in luciferase activity (Fig. 5B). These results implicate a direct role of DNA methylation of the segment containing the CpG islands 1 and 2 in transcriptional regulation of $c d k 5 r a p 1$ gene expression.

To further explore whether piR-DQ541777 directly binds to $c d k 5$ rap 1 and regulates gene expression through the methylation-dependent way, we cotransfected piR-DQ541777 and the above reporter vectors containing wild-type (wt) or mutant (mut) binding sites into SH-SY5Y cells and tested the effects of piR-DQ541777 on the luciferase activities of the reporter genes (Fig. 5C). We found that cotransfection of piR-DQ541777 with pGL-wt-C1/2 (wt1-wt2) or pGL-wt-C1 (wt1-mut2) considerably decreased luciferase activities compared with pGL-mut-C1/2 (mut1-mut2; Fig. 5D). Transfection of piR-DQ541777 also significantly decreased the luciferase activities induced by pGLwt-C2 (mut1-wt2; Fig. 5D). Moreover, administration of the demethylation agent 5 '-aza-2'-deoxycytidine (5-aza-dc) significantly reversed the decreased luciferase activity induced by 
A

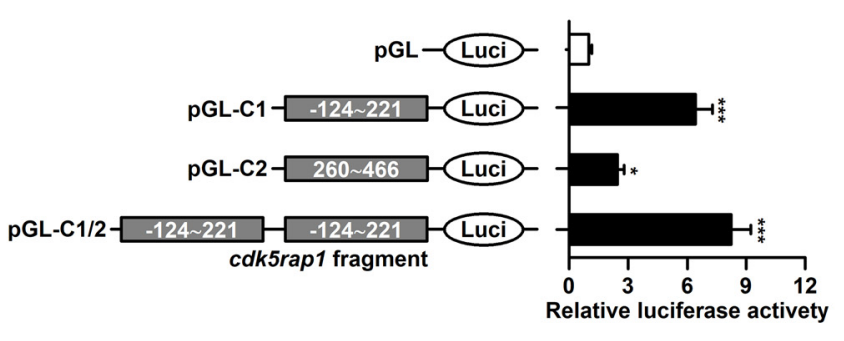

C

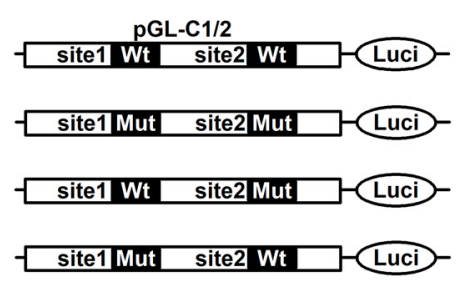

$\mathbf{F}$

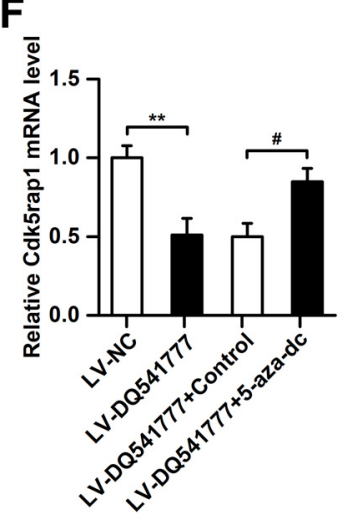

D

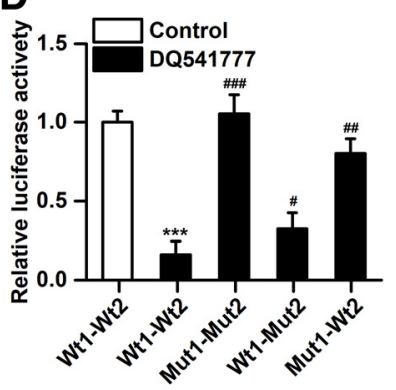

G

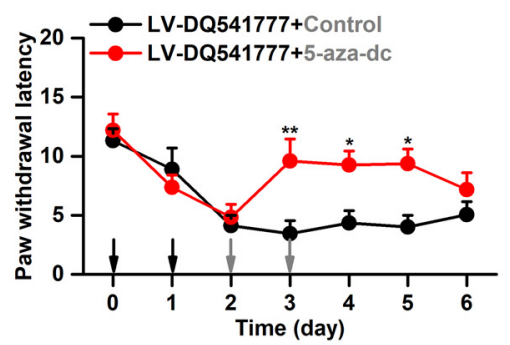

B

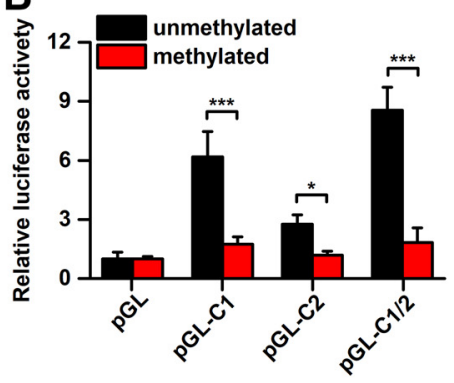

E
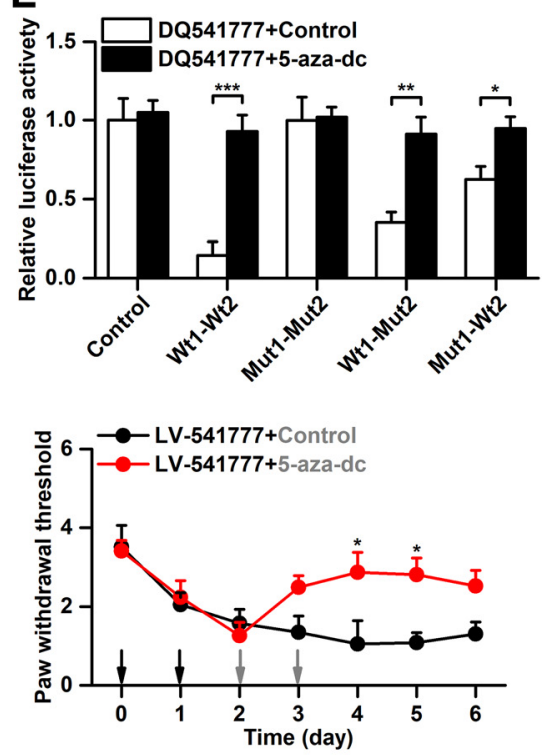

Figure 5. piR-DQ541777 regulates the Cdk5rap1 expression via methylation of its $\mathrm{CpG}$ islands. $A$, The activity of the cloned segments of $c d k 5$ rap 1 was identified by luciferase reporter assay in HEK293T cells. One-way ANOVA; $F_{(3,20)}=144.0, p<0.001 ;{ }^{*} p<0.05$, ${ }^{* * *} p<0.001$, vs pGL; $p G L C 1$, the segments of $c d k 5$ rap 1 contains CpG island 1; pGLC2, the segments of $c d k 5$ rap 1 contains CpG island 2; pGLC1/2, the segments of cdk5rap 1 contains both the CpG islands 1 and 2; $n=6$. B. The activity of methylated or demethylated segments of cdk5rap 1 encompassing CpG islands was detected by firefly luciferase reporter assays. $t_{(10)}=8.12,7.39,11.97 ;{ }^{*} p<0.05,{ }^{* * *} p<0.001, n=6 . \boldsymbol{C}, \boldsymbol{D}$, Changes of the luciferase activity after cotransfection with the above luciferase reporters and piR-DQ541777 mimic. The mutation was generated in the binding sequence between piR-DQ541777 and cdk5rap1 as indicated by pGL-wt-C1 (W1-Mut2), pGL-wt-C2 (Mut1-Wt2), and pGL-mut-C1/2 (Mut1-Mut2). One-way ANOVA; $F_{(4,25)}=106.6, p<0.001 ;{ }^{* * *} p<0.001$, vs Wt1-Wt2; \#p $<0.05$, \#\# $<0.01$, \#\#\# $<0.001$, vs Mut1-Mut2; $n=6$. E, Changes of the luciferase activity induced by cotransfection with the luciferase reporters and piR-DQ541777 mimic after treatment with 5 -aza-dc. $t_{(10)}=14.21,10.91,7.12 ;{ }^{*} p<0.05,{ }^{* *} p<0.01,{ }^{* * *} p<0.001, n=6$. $\boldsymbol{F}$, The 5-aza-dc increase the spinal Cdk5rap1 expression in LV-DQ541777-treated mice. One-way ANOVA; $F_{(3,20)}=48.53, p<0.01 ; * * p<0.01, \# p<0.05, n=6$. G, The 5-aza-dc reversed LV-DQ541777-induced thermal hyperalgesia and mechanical allodynia. Two-way ANOVA; group for PWL, $F_{(1,14)}=49.18, p<0.01 ;$ group for PWL, $F_{(1,14)}=52.94, p<0.01 ;{ }^{*} p<0.05,{ }^{* *} p<$ $0.01, n=8$.

cotransfection of piR-DQ541777 with pGL-wt-C1/2, pGL-wtC1, and pGL-wt-C2 (Fig. 5E). Last, we examined the role of DNA methylation in mediating Cdk5rap1 expression related to piRDQ541777 in vivo. We found that the decreased expression of Cdk5rap1 mRNA was reversed by intrathecal injection of 5 -aza-dc in LV-DQ541777-treated mice (Fig. 5F). In addition, spinal administration of 5-aza-dc significantly alleviated the thermal hyperalgesia and mechanical allodynia induced by the overexpression of spinal piR-DQ541777 in naive mice (Fig. 5G). Together, these results suggest that piR-DQ541777 regulates Cdk5rap1 expression via methylation of the CpG islands in the promoter region.

DNMT3a mediates the modulation of piR-DQ541777 on the methylation level of $c d k 5 \mathrm{rap} 1$

It has been reported that piRNA and methyltransferase can form a complex to participate in DNA methylation (Wu et al., 2015;
Laisné et al., 2018). Therefore, we speculate that piR-DQ541777 regulates the methylation level of the $c d k 5 r a p 1$ gene by recruiting methyltransferase to their binding sites. To verify our hypothesis, we immunoprecipitated three methyltransferases (DNMT1, DNMT3a, and DNMT3b) from control and CCI mice, and measured the level of piR-DQ541777 bound to the methyltransferase by RT-qPCR. The results showed that an increased level of piRDQ541777 bound to DNMT3a, but not DNMT1 and DNMT3b, in CCI mice compared with the sham control mice (Fig. 6A). Consistently, the interaction level between DNMT3a and MIWI protein was also increased in the CCI group versus the sham group (Fig. 6B). Next, we studied the association between DNMTs and the binding sites of piR-DQ541777 and the $c d k 5 r a p 1$ gene. We found that CCI significantly increased the level of DNMT3a bound to both site 1 and site 2 when compared with the sham-operated group. Knockdown of spinal piR-DQ541777 by intrathecal administration of sh-DQ541777 significantly inhib- 


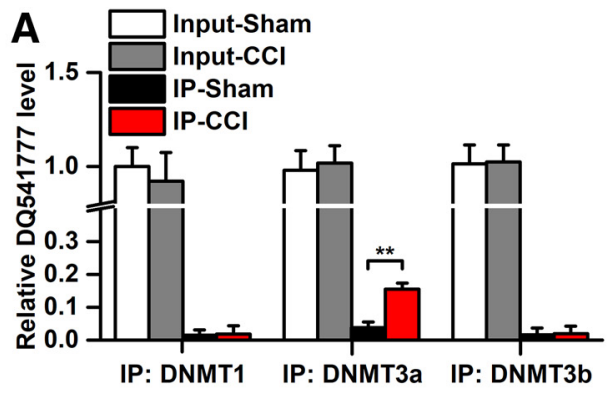

B
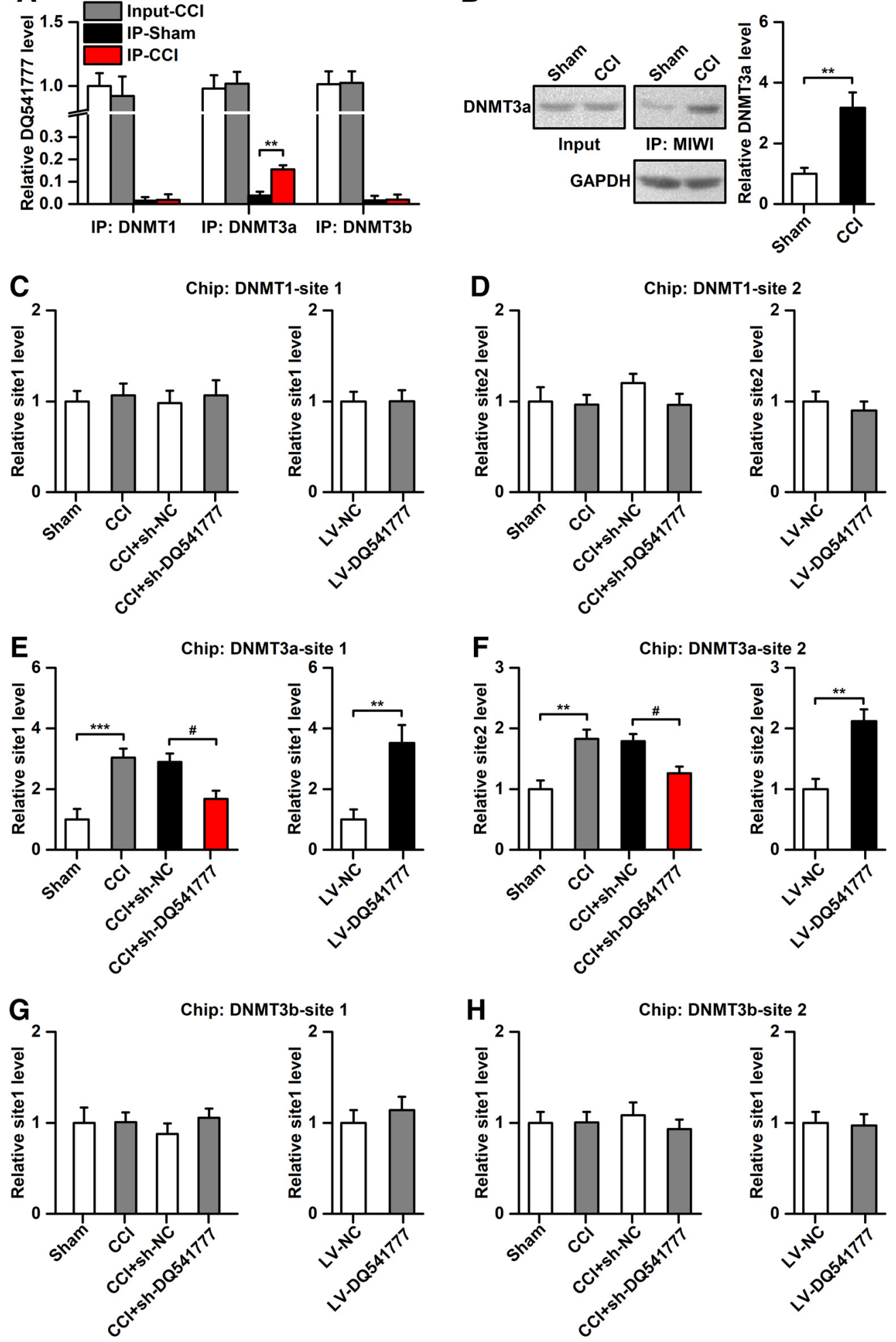

Figure 6. DNMT3a mediates the methylation of cdk5rap1 related to piR-DQ541777. $A$, The capacity of methyltransferases (DNMT1, DNMT3a, and DNMT3b) bind to piR-DQ541777 in the $\mathrm{CCl}$ group vs sham group by the RIP method. $t_{(10)}=11.57 ;{ }^{* *} p<0.01, n=6 . \boldsymbol{B}$, The interaction level of DNMT3a and MIWI after CCI by immunoprecipitation. $t_{(10)}=9.97 ;{ }^{* *} p<0.01, n=6 . C$, $D$, The change of DNMT1 binds to the sites of piR-DQ541777 and cdk5rap 1 by ChIP method. $n=6$. E, $F$, The change of DNMT3a bind to the sites of piR-DQ541777 and cdk5rap1. One-way ANOVA; $F_{(3,20)}=64.15,57.19, p<0.01 ; t_{(10)}=9.03,10.68 ;{ }^{* *} p<0.01,{ }^{* * *} p<0.001, \# p<0.05, n=6 . \mathbf{G}, \boldsymbol{H}$, The change of DNMT3b bind to the sites of piR-DQ541777 and $c d k 5 \mathrm{rap} 1 . n=6$.

ited CCI-induced high level of DNMT3a bond to the two sites (Fig. $6 E, F)$. On the contrary, overexpression of spinal piR5147777 by intrathecal administration of LV-DQ541777 significantly increased the level of the DMNT3a bond to site 1 and site 2
(Fig. 6E,F). Neither DNMT1 (Fig. 6C,D) nor DNMT3b (Fig. $6 G, H)$ show any significant difference in the level of binding to site 1 or site 2. To exclude the possibility that piR-DQ541777 may have an effect on the methyltransferase expression, we examined 

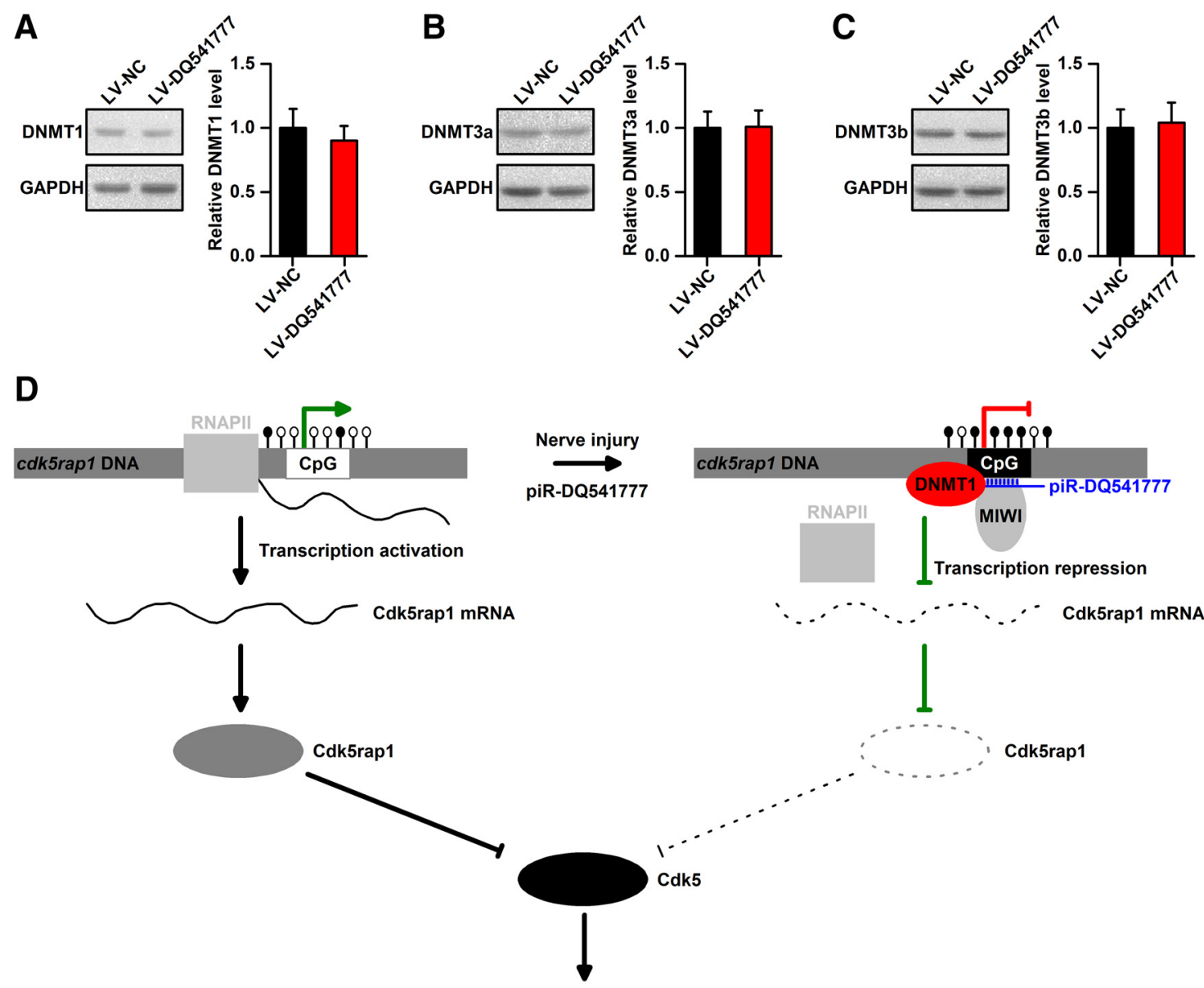

Spinal neuron sensitization

Figure 7. Effect of piR-DQ541777 on DNMT expression. $\boldsymbol{A}$, Spinal DNMT1 expression after intrathecal injection of LV-DQ541777 in naive mice. $t_{(10)}=1.29 ; n=6 . \boldsymbol{B}$, The expression of DNMT3a in the spinal cord after LV-DQ541777 injection in mice. $t_{(10)}=0.13 ; n=6$. C, The expression of spinal DNMT3b expression in LV-DQ541777-treated mice. $t_{(10)}=0.46 ; n=6$. D, Schematic of regulation of nociceptive sensitization by piR-DQ541777-mediated methylation of the cdk5rap1.

the expression of DNMT1, DNMT3a, and DNMT3b, after LVDQ541777 injection in naive mice. The results showed that overexpression of piR-DQ541777 had no significant effect on the expression of DNMT1 (Fig. 7A), DNMT3a (Fig. 7B), and DNMT3b (Fig. 7C) compared with the control group. Together, these findings suggest that piR-DQ541777 regulates the methylation level of Cdk5rap1 by recruiting DNMT3a to the promoter of $c d k 5$ rapl (Fig. $7 D$ ).

\section{Discussion}

In the present study, we found that piR-DQ541777 regulates neuropathic pain by the methylation of $c d k 5 \mathrm{rap} 1$ in the spinal cord. The major findings are as follows: (1) CCI-induced neuropathic pain significantly increased the expression of piR-DQ541777 in spinal cord; (2) manipulation of spinal piRDQ541777 expression affects pain behaviors and central sensitization in mice; (3) piR-DQ5417777 regulates CCIinduced neuropathic pain by targeting Cdk5rap1; and (4) piRDQ541777 recruits DNMT3a to the CpG islands of the cdk5rap1 promoter and as a result increases the methylation of $c d k 5$ rap 1 . Together, these results revealed an important role of piR-DQ541777 in the occurrence and development of neuropathic pain.

Spinal cord central sensitization, one of the key mechanisms for the generation and maintenance of chronic pain, largely depends on the abnormal expressions of receptor, ion channels, and intracellular signal transduction pathways in neurons (Latremoliere and Woolf, 2009; Alles and Smith, 2018). These abnormal expressions of the receptor, ion channels, and intracellular signal transduction pathways mostly result from aberrations in gene regulation. However, the mechanisms of gene expression regulation in chronic pain still remain unclear. Noncoding RNA, which was discovered recently, is an important regulator of gene expression. Emerging studies have shown that chronic pain not only causes changes in the expression of ncRNAs, such as microRNA, siRNA, and long ncRNA, but also changes in their target genes and differences in expression during the development and maintenance of chronic pain (Bali and Kuner, 2014). As a new class of ncRNA, piRNA has attracted more and more attention to its potential role in complicated biological processes. Previous studies have shown that piRNA is expressed in testicular tissues (Ro et al., 2007). However, recent evidence indicates that piRNA is also widely expressed in nervous system tissues (Lee et al., 2011; Phay et al., 2018), suggesting that it may also be involved in the regulation of the pathophysiological processes of the nervous system. In the present study, we first report that a nervous system-wide expressed piRNA, piR-DQ541777, is involved in the regulation of chronic neuropathic pain. piR-DQ541777 is expressed in spinal cord, and modulation of it significantly changes the neuropathic pain behaviors in mice. Our study has expanded the knowledge about the functional role of ncRNA, especially 
piRNA, in the generation of neuropathic pain and also deepened the understanding of the molecular mechanisms of neuropathic pain regulation. A recent study reported that 18 rat piRNAs identified in the sciatic nerve show differential changes in their levels after nerve injury, indicating that axonally localized piRNAs are critical to regulating nerve regeneration (Lee et al., 2011). Emerging evidence suggests that the active regeneration process of periphery nerves plays a pivotal role in the production and maintenance of neuropathic pain (Xie et al., 2017). Therefore, specifically targeting the piRNAs in the periphery nerve system may also provide effective pain relief. In the future, more research is needed to apply and test the role of piRNAs in pain.

CDK5 is a proline-directed serine/threonine kinase. Previous studies have shown that CDK5 is closely related to pain modulation (Pareek and Kulkarni, 2006). Chronic inflammation or peripheral nerve injury significantly increased the expression of CDK5 in the spinal cord. Knockdown of CDK5 expression or inhibition of CDK5 activity in the spinal cord attenuated pain behaviors induced by inflammation or nerve injury in mice (Fang-Hu et al., 2015; Moutal et al., 2019). Cdk5rap1 is an endogenous inhibitor of CDK5. Overexpression of Cdk5rap 1 could significantly inhibit the activity of CDK5 (Ching et al., 2002; Liu et al., 2018). Although many studies have reported that CDK5 is involved in pain regulation, few studies reported the connection between Cdk5rap1 and pain. Here, our findings revealed that the expression of spinal Cdk5rap 1 was significantly reduced in CCI-induced neuropathic pain mice. Moreover, this reduction of Cdk5rap1 contributed to the modulation of neuropathic pain related to spinal piR-DQ541777. Together, we propose that Cdk5rap1 is also a key player in the development of neuropathic pain. Although there is a clear association between the effect of piR-DQ541777 on pain and piRDQ541777 on Cdk5rap1, no formal proof for causality is the limitation of the present study.

piRNA exerts its function through a wide variety of molecular mechanisms. In animal germ lines, piRNA interacts with the Piwi proteins to maintain genome integrity by silencing transposons (Aravin et al., 2007). piR-ABC interacts with the 3' UTR of the tnfs4 gene and controls the expression of TNFSF4 in bladder cancer (Chu et al., 2015). In addition, recent evidence suggested that piRNA also showed an important role in epigenetic regulation of gene expression. Heterochromatin protein 1a interacted with the piRNA-Piwi complex and is required for the histone modification (Iwasaki et al., 2016). The piRNA-Piwi complex regulates the gene expression by direct methylation of histone $\mathrm{H} 3$ lysine 9 in somatic cells in the adult Drosophila ovary (Lin and Yin, 2008). In the present experiment, our results also showed that piRNA controls its target gene expression through an epigenetic mechanism. Two CpG islands were found near the binding sites between piR-DQ541777 and cdk5rap1. In vitro and in vivo modulation of piR-DQ541777 significantly affect the methylation level of both the $\mathrm{CpG}$ islands in the $c d k 5$ rap 1 promoter. Furthermore, we found that DNMT3a could be recruited by piRDQ541777-MIWI complex and transferred to the binding sites between piR-DQ541777 and $c d k 5$ rap1. These results suggest that the epigenetic mechanism mediates the regulation of neuropathic pain related to spinal piR-DQ541777.

In conclusion, this study has improved our understanding of piRNA in the physiology and pathology of the nervous system and elucidates a new molecular mechanism of neuropathic pain.
Our findings may lead to novel drug target discovery for the prevention and treatment of neuropathic pain.

\section{References}

Alles SRA, Smith PA (2018) Etiology and pharmacology of neuropathic pain. Pharmacol Rev 70:315-347.

Aravin AA, Sachidanandam R, Girard A, Fejes-Toth K, Hannon GJ (2007) Developmentally regulated piRNA clusters implicate MILI in transposon control. Science 316:744-747.

Bali KK, Kuner R (2014) Noncoding RNAs: key molecules in understanding and treating pain. Trends Mol Med 20:437-448.

Bennett GJ, Xie YK (1988) A peripheral mononeuropathy in rat that produces disorders of pain sensation like those seen in man. Pain 33:87-107.

Chaplan SR, Bach FW, Pogrel JW, Chung JM, Yaksh TL (1994) Quantitative assessment of tactile allodynia in the rat paw. J Neurosci Methods 53:5563.

Ching YP, Pang AS, Lam WH, Qi RZ, Wang JH (2002) Identification of a neuronal Cdk5 activator-binding protein as Cdk5 inhibitor. J Biol Chem 277:15237-15240.

Chu H, Hui G, Yuan L, Shi D, Wang Y, Du M, Zhong D, Ma L, Tong N, Qin C, Yin C, Zhang Z, Wang M (2015) Identification of novel piRNAs in bladder cancer. Cancer Lett 356:561-567.

Decosterd I, Woolf CJ (2000) Spared nerve injury: an animal model of persistent peripheral neuropathic pain. Pain 87:149-158.

Esteller M (2011) Non-coding RNAs in human disease. Nat Rev Genet 12 : 861-874.

Fang-Hu, Zhang HH, Yang BX, Huang JL, Shun JL, Kong FJ, Peng-Xu, Chen ZG, Lu JM (2015) Cdk5 contributes to inflammation-induced thermal hyperalgesia mediated by the $\mathrm{p} 38$ MAPK pathway in microglia. Brain Res 1619:166-175.

Gao YJ, Ji RR (2009) c-fos and pERK, which is a better marker for neuronal activation and central sensitization after noxious stimulation and tissue injury? Open Pain J 2:11-17.

Hargreaves K, Dubner R, Brown F, Flores C, Joris J (1988) A new and sensitive method for measuring thermal nociception in cutaneous hyperalgesia. Pain 32:77-88.

Holoch D, Moazed D (2015) RNA-mediated epigenetic regulation of gene expression. Nat Rev Genet 16:71-84.

Iwasaki YW, Siomi MC, Siomi H (2015) PIWI-interacting RNA: its biogenesis and functions. Annu Rev Biochem 84:405-433.

Iwasaki YW, Murano K, Ishizu H, Shibuya A, Iyoda Y, Siomi MC, Siomi H, Saito K (2016) Piwi modulates chromatin accessibility by regulating multiple factors including histone $\mathrm{H} 1$ to repress transposons. Mol Cell 63:408-419.

Kim SH, Chung JM (1992) An experimental model for peripheral neuropathy produced by segmental spinal nerve ligation in the rat. Pain 50: 355-363.

Kuner R, Flor H (2016) Structural plasticity and reorganisation in chronic pain. Nat Rev Neurosci 18:20-30.

Laisné M, Gupta N, Kirsh O, Pradhan S, Defossez PA (2018) Mechanisms of DNA methyltransferase recruitment in mammals. Genes (Basel) 9:E617.

Latremoliere A, Woolf CJ (2009) Central sensitization: a generator of pain hypersensitivity by central neural plasticity. J Pain 10:895-926.

Lee EJ, Banerjee S, Zhou H, Jammalamadaka A, Arcila M, Manjunath BS, Kosik KS (2011) Identification of piRNAs in the central nervous system. RNA 17:1090-1099.

Lin H, Yin H (2008) A novel epigenetic mechanism in Drosophila somatic cells mediated by Piwi and piRNAs. Cold Spring Harb Symp Quant Biol 73:273-281

Liu KC, Leuckx G, Sakano D, Seymour PA, Mattsson CL, Rautio L, Staels W, Verdonck Y, Serup P, Kume S, Heimberg H, Andersson O (2018) Inhibition of Cdk5 promotes beta-cell differentiation from ductal progenitors. Diabetes 67:58-70.

Livak KJ, Schmittgen TD (2001) Analysis of relative gene expression data using real-time quantitative PCR and the $2(-\delta \delta \mathrm{C}(\mathrm{T}))$ method. Methods 25:402-408.

Moutal A, Luo S, Largent-Milnes TM, Vanderah TW, Khanna R (2019) Cdk5-mediated CRMP2 phosphorylation is necessary and sufficient for peripheral neuropathic pain. Neurobiol Pain 5:100022.

Ozata DM, Gainetdinov I, Zoch A, O'Carroll D, Zamore PD (2019) PIWIinteracting RNAs: small RNAs with big functions. Nat Rev Genet 20: 89-108. 
Pareek TK, Kulkarni AB (2006) Cdk5: a new player in pain signaling. Cell Cycle 5:585-588.

Phay M, Kim HH, Yoo S (2018) Analysis of piRNA-like small non-coding RNAs present in axons of adult sensory neurons. Mol Neurobiol 55: 483-494.

Rajasethupathy P, Antonov I, Sheridan R, Frey S, Sander C, Tuschl T, Kandel ER (2012) A role for neuronal piRNAs in the epigenetic control of memory-related synaptic plasticity. Cell 149:693-707.

Ro S, Park C, Song R, Nguyen D, Jin J, Sanders KM, McCarrey JR, Yan W (2007) Cloning and expression profiling of testis-expressed piRNA-like RNAs. RNA 13:1693-1702.

Vagin VV, Sigova A, Li C, Seitz H, Gvozdev V, Zamore PD (2006) A distinct small RNA pathway silences selfish genetic elements in the germline. Science 313:320-324.

Wu D, Fu H, Zhou H, Su J, Zhang F, Shen J (2015) Effects of novel ncRNA molecules, p15-piRNAs, on the methylation of DNA and histone $\mathrm{H} 3$ of the CDKN2B promoter region in U937 cells. J Cell Biochem 116: $2744-2754$

Xie W, Strong JA, Zhang JM (2017) Active nerve regeneration with failed target reinnervation drives persistent neuropathic pain. eNeuro 4: ENEURO.0008-17.2017.

Zhou X, Zhang C, Zhang C, Peng Y, Wang Y, Xu H (2017) MicroRNA$182-5 p$ regulates nerve injury-induced nociceptive hypersensitivity by targeting ephrin type-b receptor 1. Anesthesiology 126:967-977. 